\section{Differential diagnosis of isolated myeloid sarcoma: a case report and review of the literature}

\author{
Patrick A. Hagen, ${ }^{1}$ Charanjeet Singh, ${ }^{2}$ \\ Melissa Hart, ${ }^{2}$ Anne H. Blaes ${ }^{1}$ \\ 1Division of Hematology/Oncology/ \\ Transplantation, Department of Medicine; \\ 2Division of Pathology, University of \\ Minneapolis, MN, USA
}

\begin{abstract}
Myeloid sarcoma (MS) is a rare disease entity identified as a variety of manifestations defined by the occurrence of extramedullary myeloid cell masses with or without bone marrow involvement. This case describes an unusual presentation of isolated MS in a 60-year-old otherwise healthy male, who initially presented to his primary care physician with vague abdominal pain. After extensive workup including three omental biopsies, umbilical core biopsy, and inguinal lymph node biopsy, he was ultimately diagnosed with isolated MS with extensive extramedullary tumor burden. Despite advanced extramedullary disease, peripheral cell counts were normal and bilateral bone marrow biopsies unremarkable with normal cellular lineages, morphology, and cytogenetics. The patient underwent induction chemotherapy and is now greater than 100 days post myeloablative unrelated donor marrow transplantation with no evidence of disease recurrence and $100 \%$ donor status with full chimerism. This case demonstrates that making a prompt diagnosis with rapid initiation of treatment in myeloid sarcoma can be challenging due to its varied clinical presentation, cytomorphology, cytochemistry, and cytogenetic overlap with other lymphoid malignancies. Once a diagnosis of MS has been made, moving quickly to induction therapy is important. Several studies have shown that improved overall survival is attained when MS is treated as acute myeloid leukemia and increased survival is noted for patients undergoing bone marrow transplantation. Further prospective studies are needed to elucidate the many remaining questions in regards to the natural history, prognosis, and optimal treatment strategies for this deadly disease.
\end{abstract}

\section{Introduction}

Myeloid sarcoma (MS) is a rare disease entity defined by the occurrence of one or more extramedullary myeloid cell masses with or without bone marrow involvement. ${ }^{1}$ It was first described in $1812,{ }^{2}$ and later termed a chloroma by King, ${ }^{3}$ because of its green appearance on gross morphology owing to myeloperoxidase enzymes in the myeloblasts. Since it was first recognized, knowledge of its varied presentation, association with acute myeloid leukemia (AML) and other myeloproliferative disorders, means of diagnosis, and preferred treatment approach has evolved. Here, we report a case of isolated MS with unusually extensive extramedullary tumor burden. This case and literature review illustrate the challenges of making a prompt diagnosis and elucidates a diagnostic approach to ensure rapid initiation of treatment. Written informed consent was obtained from the patient for publication of this case report and any accompanying images.

\section{Case Report}

A 60-year-old male presented to his primary care physician with vague abdominal pain of one-month duration. A hardened umbilicus was noted on physical exam. The patient was initially misdiagnosed with an umbilical hernia and subsequently peri-umbilical cellulitis. The abdominal pain intensified and required hospital admission. An abdominal computed tomography (CT) scan showed signs of peritoneal carcinomatosis and liver lesions. A colonoscopy was performed to screen for possible metastatic colorectal cancer; however, no notable masses or polyps were detected. Laboratory workup was largely unremarkable showing a white blood cell count of $5.610^{9} / \mathrm{L}$, hemoglobin of $15.5 \mathrm{~g} / \mathrm{dL}$, and platelets of $281 \times 10^{9} / \mathrm{L}$. Basic metabolic panel, liver function tests, uric acid, immunoglobulins, and serum protein electrophoresis were all within normal limits except for an elevated creatinine of $1.38 \mathrm{mg} / \mathrm{dL}$ and lactate dehydrogenase of $1541 \mathrm{U} / \mathrm{L}$ (normal range 325-750). A CT guided core needle biopsy of a likely omental mass was obtained showing an infiltrate of atypical cells positive for CD43, CD117, and CD99 with a subset of cells positive for CD68 and myeloperoxidase (MPO). Because of limited tissue, however, additional immunophenotyping and cytogenetic analysis was not performed to definitively classify the CD68+/MP0+ lineage. On the basis of these findings, the patient was referred to our outpatient oncology clinic two months after the initial presentation of abdominal pain.

Aleukemic leukemia with myelocytic sarcoma was suspected during outpatient oncology follow-up. Bilateral bone marrow biopsies of the iliac spine showed normal cellular lineages, iron findings, morphology, and cytogenet-
Correspondence: Patrick A. Hagen, Division of Internal Medicine, University of Minnesota, 1229 Oxford Rd, Deerfield, IL 60015, USA.

Tel.: +1.847.997.3626 - Fax: +1.708.327.3319.

E-mail: hagen657@umn.edu

Key words: Myeloid; sarcoma; leukemia.

Contributions: the authors contributed equally.

Conflict of interest: the authors declare no potential conflict of interest.

Received for publication: 7 November 2014 .

Revision received: 9 March 2015.

Accepted for publication: 16 April 2015.

This work is licensed under a Creative Commons Attribution NonCommercial 3.0 License (CC BYNC 3.0).

(C) Copyright P.A. Hagen et al., 2015

Licensee PAGEPress, Italy

Hematology Reports 2015; 7:5709

doi:10.4081/hr.2015.5709

ics. To obtain additional tissue for definitive immunophenotyping and cytogenetics, a minimally invasive umbilical core biopsy of the hardened umbilicus and a fine needle aspiration of an enlarged inguinal lymph node noted on previous CT imaging were performed. The umbilical core biopsy stained positive for CD68-KP1, CD117, and MP0 and negative for CD34 and CD56; this immunophenotypic profile is consistent with MS. However, due to extensive crush artifact, additional cytogenetics and immunophenotyping were unable to be performed. The inguinal lymph node biopsy was unremarkable. A positron emission tomography CT (PET-CT) scan showed advanced metastatic disease throughout the chest, abdomen, and pelvis with multiple hypermetabolic lymph nodes. Advanced pleural/omental caking was observed along with a $7.7 \times 6.1 \mathrm{~cm}$ mass at the root of the mesentery invading the adjacent small intestine. Upon readmission for planned induction chemotherapy, a final laparoscopic omental biopsy was performed with tissue adequate to perform the necessary studies needed to make a definitive diagnosis of lymphoma versus MS. Myeloid cell markers including CD99, CD117, CD68-KP1, CD43, MP0, and lysozyme were evident from the omental and umbilical biopsies; however, T and $B$ cell markers were absent. These findings differentiated MS from B and T cell lymphomas and poorly differentiated carcinoma. Representative stains of important myeloid markers as well as the aggressive nature of this myeloproliferative disease with tumor infiltrating fat are shown in Figure 1. Molecular abnormities frequently encountered in AML including FLT3-ITD and NPM1-mutant 
were found. On the basis of these observations, a definitive diagnosis was made of MS 46XY, FLT3-ITD and NPM1-mutant involving the peritoneum and pleural cavity. The patient underwent $7+3$ induction chemotherapy with idarubicin and cytarabine followed by two cycles of consolidation with intermediate-dose cytarabine. The patient then received myeloablative unrelated donor bone marrow transplantation. Follow-up PET-CT scan and bone marrow biopsy at 100 days post-transplantation showed no evidence of disease recurrence and $100 \%$ donor status with full chimerism.

\section{Discussion}

The differential diagnosis of MS is often a clinical challenge. Menasce $e t$ al. published a review of 26 cases of MS in which 15 of the 26 were misdiagnosed mostly as diffuse large Bcell lymphoma. ${ }^{4}$ Furthermore, Byrd et al. examined all known MS cases in the literature as of 1995 and found that of the 154 published isolated MS cases, $46 \%$ had initial erroneous diagnoses often mislabeled as large cell lymphoma..$^{5}$ Mature or immature types of MS can be confused with Hodgkin lymphoma, T-cell lymphomas, extramedullary hematopoiesis (myeloid metaplasia), or infectious processes. Additionally, blastic types of MS can be readily confused with non-Hodgkin lymphoma, lymphoblastic lymphoma, poorly differentiated carcinoma, or melanoma. ${ }^{6}$ Particularly challenging is differentiating MS from lymphoma as MS frequently stains with T-cell markers (CD43, CD45, or CD3) and variably stains with B-cell markers (CD79a). ${ }^{7}$ For these reasons, accurate diagnosis and sub-classification of MS requires correlation of morphology, immunophenotype, cytogenetics, and molecular diagnostics (please refer to Table 1). Although no definitive diagnostic steps can be applied to all clinical scenarios, an understand- ing of the typical clinical presentation and necessary workup is useful in reaching an accurate and prompt diagnosis.

Myeloid sarcoma is most commonly associated with AML. The proportion of AML cases that are associated with MS varies across studies, but usually ranges from $3-10 \% .^{7-10}$ MS can also be present in the context of other myeloproliferative disorders, such as chronic myelogenous leukemia, multiple myeloma, myelodysplastic syndrome, or myelofibrosis. When MS co-occurs with another hematological malignancy, a diagnosis is more likely to be obtained. In contrast, isolated or primary MS is more difficult to diagnosis even in the setting of a large tumor burden as in our case. The most common sites of presentation include the skin, lymph node, soft tissue, and bone; $5,7,11-13$ however, nearly all anatomical locations have been described including the ovaries, myocardium, pineal body, and other locations. Most patients who are diagnosed with isolated MS develop traditional AML with bone marrow involvement within five to nine months, but some develop AML up to 9 years after the initial MS diagnosis. 8,14

In making a diagnosis of MS, histopathology and cytomorphology are less informative than cytogenetics, cytochemistry, and immunophenotyping. Audouin et al. noted that the infiltrate in MS can be massive, obscuring and destroying normal tissue, with perivascular infiltration being a common histopathologic finding. ${ }^{15}$ Cytomorphologic classification of tumors by degree of differentiation does not change the prognosis of the patient and is clinically insignificant. ${ }^{4,16}$ While it was previously thought that the presence of eosinophilic myelocytes was a means to a definitive diagnosis, this test is much less sensitive than originally thought and should not be used as a hallmark of disease. ${ }^{4,6,16}$

The role of cytogenetics in the diagnosis of MS is becoming increasingly important. Pileri et al..$^{17}$ found that monosomy 7 (10.8\%), trisomy
$8(10.4 \%)$, and mixed lineage leukemia splitting (8.5\%) were the most frequently encountered abnormalities. The $t(8 ; 21)(p 22 ; q 22)$ translocation, previously thought to be the most common cytogenetic abnormality, is now known to be more common in childhood or in MS involving the orbit. ${ }^{5,18}$ Overall, the recorded incidence of chromosomal aberrations appears to be in line with that seen in adult AML. ${ }^{18}$

Cytochemical and immunophenotypic analysis are essential for a correct MS diagnosis. Importantly, while myelocytic markers on cytochemical analysis can aide in making a diagnosis, there absence does not exclude MS. Anti-myeloperoxidase has been shown to be the most useful and sensitive cytochemical marker, but will be absent in poorly differentiated myeloblasts. ${ }^{16}$ Lysozyme and esterase present later in the progression of MS and can be absent from poorly differentiated MS. Immunophenotyping is the most important means of making a definitive diagnosis. CD68/KP1 and CD43 are the most commonly encountered makers, both present in $75-100 \%$ of cases, ${ }^{15-19}$ but the non-specificity of CD43 in particular should be noted. Other common markers include CD117, CD99, CD34, CD56, tdt, CD61/linker of activated T lymphocyte/von Willebrand antigen, glycophorin A, and CD4. ${ }^{19}$ ${ }^{21}$ Markers of myeloid differentiation include MPO, KPI, and CD117.7,16,20,21 Conversely, markers important for monocytic differentiation are CD68, CD163, and CD56. ${ }^{7,19,20}$ Cutaneous MS is frequently monocytic in origin and negative for MPO and CD117. ${ }^{19}$ There are several markers that are not indicative of MS and can lead to misdiagnoses. CD30 is rarely expressed in MS and cannot be relied upon as a myeloid differentiator. ${ }^{17}$

Differentiating MS from lymphoma is challenging. MS will often express aberrant $\mathrm{B}$ or T cell makers. Leukocyte common antigen (LCA) and CD43 can be expressed in MS, and if the non-specificity of CD43 is not recognized and other myeloid markers are absent, an incorrect

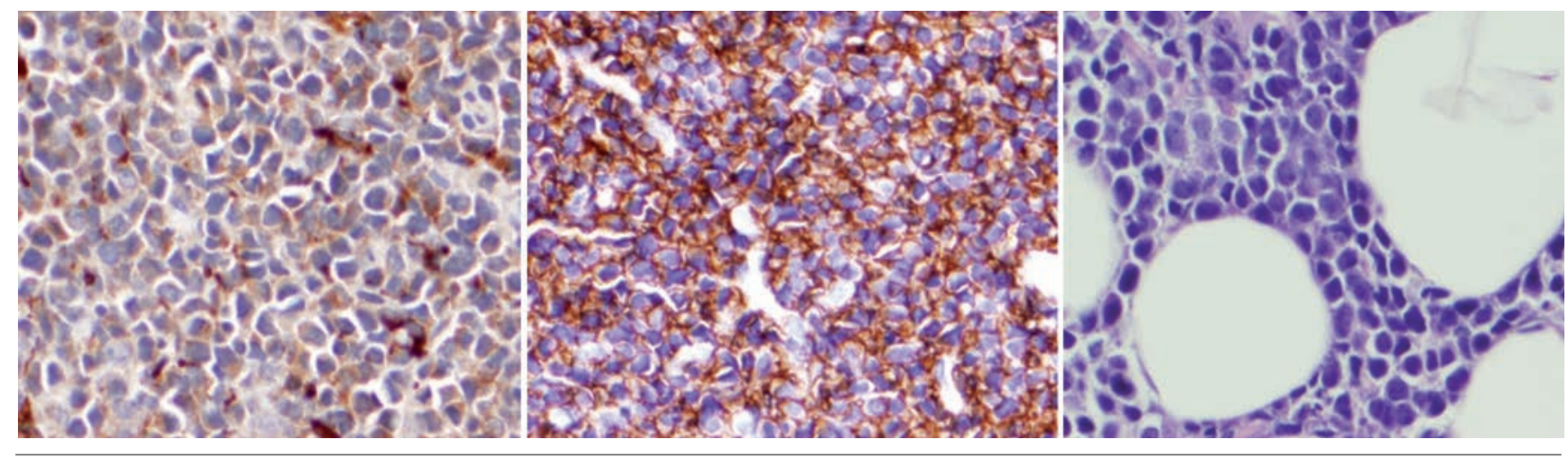

Figure 1. Representative images of omental tissue sections stained for CD43 (left panel), CD68 (middle panel), and high power Hematoxylin \& Eosin showing tumor infiltrating fat (right panel). 
diagnosis of T-cell lymphoma can be reached. ${ }^{4,15}$ Additionally, CD15 (Hodgkin lymphoma) and CD20 (B-cell lymphoma) are not useful to differentiate MS from lymphoma because both markers can be expressed in MS and lymphoma. ${ }^{16}$ Importantly, induction therapy for the commonly misdiagnosed diseases including $B$ and $T$ cell lymphomas are different than that for MS, and when the diagnosis is delayed or missed altogether, a clinical disservice is done to the patient.

\section{Conclusions}

Table 1 offers our guidelines and approach in making a diagnosis of MS. This diagnosis is often delayed because of the variance with which the disease can present clinically. This was evident in our case as the patient was seen by two clinicians with two separate nonhematological diagnoses considered prior to the patient's worsening visceral symptoms leading to PET-CT imaging and an MS diagnosis. The importance of recognizing the wide array of possible clinical presentations as well as the necessity for adequate cellular studies cannot be overstated if one is to optimally treat this rare clinical condition. Once a diagnosis of MS has been made, moving quickly to induction therapy is important because median survival is quite poor ranging from 1-2 years but as low as 8 months. ${ }^{1,8,11,13,14,17,22,23}$ While studies have generally shown that MS is not consistently influenced by sex, anatomical site, primary versus secondary MS, or phenotyping/cytogenetic findings, ${ }^{9,14,17}$ the recent publication by Movassaghian et al. may challenge this. ${ }^{13}$ After reviewing the SEER database from 1973 to 2010, they found 345 cases of isolated MS. Among these, isolated MS was more favorable than AML without MS and also found that MS of the soft tissue, lymph node, or nervous system all carry a worse prognosis. Additional unfavorable prognostic indicators in the literature appear to include leukemia cutis and MS in association with myeloproliferative disorders other than AML. 7,14,24

Regardless of the prognosis, several studies have shown that improved overall survival is attained when MS is treated as per AML. Indeed, increased survival is noted for patients undergoing allogeneic bone marrow transplantation. 9,17 Our case demonstrates that when a diagnosis can be promptly made and the patient responds well to induction chemotherapy, allogeneic bone marrow transplantation may offer patients a more favorable long-term prognosis. Further prospective studies are needed to elucidate the many remaining questions in regards to the natural history, prognosis, and optimal treatment strategies for this deadly disease.

Table 1. Guidelines for diagnosing myeloid sarcoma (MS).

\begin{tabular}{|c|c|}
\hline Differential diagnosis & $\begin{array}{l}\text { - Mature or immature types of MS: Hodgkin lymphoma, T-cell lymphoma; } \\
\text { extramedullary hematopoiesis (myeloid metaplasia), or infectious } \\
\text { processes; } \\
\text { - Blastic type of MS: non-Hodgkin's lymphoma, lymphoblastic lymphoma; } \\
\text { poorly differentiated carcinoma, melanoma }\end{array}$ \\
\hline Anatomical locations & Varied \\
\hline $\begin{array}{l}\text { Associated hematological } \\
\text { malignancies }\end{array}$ & $\begin{array}{l}\text { Acute myelogenous leukemia; chronic myelogenous leukemia; multiple } \\
\text { myeloma; myelodysplastic syndrome; myelofibrosis }\end{array}$ \\
\hline Histology (varied) & $\begin{array}{l}\text { Mature and immature myelocytes; blasts; lack of bileneage or trileneage } \\
\text { differentiation; extensive infiltration of surrounding tissue, or quite distinct }\end{array}$ \\
\hline $\begin{array}{l}\text { Cytochemistry: } \\
\text { recommended stains }\end{array}$ & $\begin{array}{l}\text { Myeloperoxidase; lysozyme; naphthol AS-D chloroacetate esterase; } \\
\text { non-specific esterase }\end{array}$ \\
\hline $\begin{array}{l}\text { Immunophenotyping: } \\
\text { recommended markers }\end{array}$ & $\begin{array}{l}\text { - Most common: CD43 and CD68/KP1 } \\
\text { - Other common: CD4, CD15, CD30, CD34, CD56, CD99, CD117, tdt, } \\
\text { Glycophorin A, CD61/linker of activated T-lymphocyte/von-Willebrand antigen } \\
\text { - Myeloid markers: CD68/KP1, CD117 } \\
\text { - Monocytic markers: CD56, CD68, CD163 } \\
\text { - T-cell markers: CD3, CD4, CD43, CD45, LCA } \\
\text { - B-cell markers: CD20, CD79a }\end{array}$ \\
\hline $\begin{array}{l}\text { Cytogenetics: } \\
\text { recommended evaluations } \\
\text { should include }\end{array}$ & $\begin{array}{l}\text { - Evaluate for monosomies, trisomies, translocations, and inversions: } \\
\text { monosomy 7, monosomy } 16 \text {, trisomy } 8 \text {, trisomy } 11, \mathrm{t}(8 ; 21)(\mathrm{p} 22 ; q 22) \text {, } \\
\text { inversion } 16 \\
\text { - Particular deletions: } 16 \mathrm{q}, 5 \mathrm{q}, 20 \mathrm{q} \\
\text { - Mutations commonly associated with acute myeloid leukemia: } \\
\text { NPM1, FLT3-ITD }\end{array}$ \\
\hline
\end{tabular}

\section{References}

1. Breccia M, Alimena G. Isolated myeloid sarcoma without BM involvement. Clin Adv Hematol Oncol 2012;10:66-7.

2. Burns A. Observations of surgical anatomy, head and neck. Edinburgh: Thomas Royce and Co; 1811. pp 364-366.

3. King A. A case of chloroma. Monthly J Med 1853;17:97.

4. Menasce LP, Banerjee SS, Beckett E, et al. Extramedullary myeloid tumor (granulocytic sarcoma) is often misdiagnosed: a study of 26 cases. Histopathology 1999;23: 391-8.

5. Byrd JC, Edenfield WJ, Shields DJ, et al. Extramudullary myeloid cell tumors in acute non-lymphocytic leukemia: a clinical review. J Clin Oncol 1995;13:1800-16.

6. Suh YK, Shin HJ. Fine-needle aspiration biopsy of granulocytic sarcoma a clinicopathologic study of 27 cases. Cancer 2000; 90:364-72.

7. Paydas S, Zorludemir S, Ergin M. Granulocytic sarcoma: 32 cases and review of the literature. Leuk Lymphoma 2006;47: 2527-41.

8. Breccia M, Mandelli F, Petti MC, et al. Clinico-pathological characteristics of myeloid sarcoma at diagnosis and during follow-up: report of 12 cases from a single institution. Leuk Res 2004;28:1165-9.

9. Avni B, Rund D, Levin M, et al. Clinical implications of acute myeloid leukemia presenting as myeloid sarcoma. Hematol Oncol 2012;30:34-40.

10. Shimizu H, Saitoh T, Hatsumi N, et al. Clinical significance of granulocytic sarcoma in adult patients with acute myeloid leukemia. Cancer Sci 2012;103:1513-7.

11. Tsimberidou AM, Kantarjian HM, Estey E. Outcome in patients with nonleukemic granulocytic sarcoma treated with chemotherapy with or without radiotherapy. Leukemia 2003;17:1100-3.

12. Kamauchi K, Yasuda M. Comparison in treatments of non-leukemic granulocytic sarcoma. Cancer 2002;94:1739-46.

13. Movassaghian M, Brunner AM, Blonquist TM, et al. Presentation and outcome among patients with isolated myeloid sarcoma: a suveillance, epidemiology, and end results database analysis. Leuk Lymphoma. 2014 Oct 9:1-6. [Epub ahead of print]

14. Neiman RS, Barcos M, Berard C, et al. Granulocytic sarcoma: a clinicopathologic study of 61 biopsied cases. Cancer 1981;48:1426-37.

15. Audouin J, Comperat E, Le Tourneau A, et al. Myeloid sarcoma: clinical and morphological criteria useful for diagnoses. Int $\mathbf{J}$ Surg Pathol 2003;11:271-82.

16. Traweek S, Arber DA, Rappaport H, et al. Extramedullary myeloid tumors. An immunohistochemical and morphologic study of 28 cases. Am J Surg Pathol 1993; 17:1011-9.

17. Pileri SA, Ascani S, Cox MC, et al. Myeloid 
sarcoma: clinico-pathologic, phenotypic and cytogenetic analysis of 92 adult patients. Leukemia 2007;21:340-50.

18. Tallman MS, Hakiimian D, Shaw JM, et al. Granulocytic sarcoma is associated with 8;21 translocation in AML. J Clin Oncol 1993;11:690-7.

19. Klco JM, Welch JS, Nguyen TT, et al. State of the art of myeloid sarcoma. Int $\mathrm{J} \mathrm{Lab}$ Hematol 2011;33:555-65.
20. Alexiev BA, Wang W, Ning Y, et al. Myeloid sarcomas: a histologic, immunohistochemical, and cytogenetic study. Diagn Pathol 2007;2:42.

21. Campidelli C, Agostinelli C, Stitson R, et al. Myeloid sarcoma: extramedullary manifestation of myeloid disorders. Am J Clin Pathol 2009; 132:426-437.

22. Imrie KR, Kovacs MJ, Selby D, et al. Isolated chloroma: the effect of early antileukemic therapy. Ann Intern Med 1995;123:351-3.

23. Tsimberidou AM, Kantarjian H, Wen S, et al. Myeloid sarcoma is associated with superior event-free survival and overall survival compared with acute myeloid leukemia. Cancer 2008;113:1370-8.

24. Zhou L, Landa C, Zaiden R. An unusual case of disseminated lymphadenopathy. Clin Adv Hematol Oncol 2012;10:64-5. 